\title{
Thermal Expansivity of Ternary Liquid Mixtures: Application of Hard-Sphere Models and Flory's Statistical Theory
}

\author{
Anjali Awasthi, B.S. Tripathi and Aashees Awasthi* \\ Department of Physics, Lucknow University, Lucknow, India
}

(Received July 14, 2009; revised version February 26, 2010; in final form July 15, 2010)

\begin{abstract}
Seven hard-sphere models and Flory's statistical mechanical theory have been applied to evaluate thermal expansion coefficient of ternary liquid systems involving dimethyl sulfoxide with phenol/o-cresol in carbon tetrachloride at $293.15,303.15$ and $313.15 \mathrm{~K}$. The results thus obtained are compared with the experimental values of thermal expansivity. The relative applicability of all these approaches to the present investigation has been checked and discussed. The excess values of thermal expansivity have also been calculated and utilized to study the presence and strength of intermolecular interactions in the ternary liquid systems under investigation.
\end{abstract}

PACS numbers: 65.40.De, 65.60.+a

\section{Introduction}

The system consisting of hard-sphere molecules is a convenient model for the initial study of the nature of dense gases and liquids. The hard-sphere (HS) system is defined by a potential of interaction considering only the repulsive forces among the molecules. The simplicity of this model allows us to calculate the thermodynamic properties by obtaining analytical solutions of theories or by performing computer simulations. A realistic approach has been provided by rigid-sphere equation of state to discuss the phenomenon of repulsive forces in simple fluids [1-5]. Thiele [1] and Wertheim [2] have profounded the closed form solution of the Percus-Yevick equation for rigid hard spheres. Reiss et al. [3] came to similar results using the scaled-particle theory. The hard-sphere model of binary liquid mixtures was proposed by Snider and Herrington [6] and they used it to calculate the excess thermodynamic functions. Mulero et al. [7] reviewed the accuracy of twenty two equations of state for hard spheres in order to perform their applications. Bomont and Bretonnet [8] used a consistent integral equation theory for hard sphere to calculate thermodynamic properties. Miandehy et al. [9] proposed a new equation of state which is extended to mixtures of hard spheres and excess functions of various binary liquid mixtures are calculated using the perturbation theory.

In recent years, Flory's statistical theory [10-13] was successfully applied to binary and multicomponent liquid mixtures for estimating various thermodynamic and transport properties. Pandey et al. [14] applied Flory's statistical theory with modified expression of characteristic pressure to discuss the thermodynamic behaviour of ternary liquid mixtures. Gepert et al. [15] provided a comparative study of Flory's model and Prigogine (PFP)

* corresponding author; e-mail: aasheesawasthi@rediffmail.com model for studying the thermodynamic properties of selected binary mixtures of hydrocarbons. Oswal et al. [16] examined and critically discussed the applicability of the ERAS and Flory models and Rao theory for the alkyl amines.

The expansivity coefficients are related to the fluctuations of the cross-term of enthalpic interaction and interaction volume of liquids and liquid mixtures. The excess amount may reflect the molecular orientation and packing of mixtures. Thermal expansivity has been widely used to compute various thermodynamic parameters. Very little work has been done in the estimation of thermal expansivity of ternary liquid mixtures. Sharma [17-19] used the expansivity data for calculating various thermo-acoustical parameters in polymers, liquified gases and some organic liquids. According to Sharma, expansivity is the controlling factor in several thermo-acoustical parameters. Tabhane et al. [20] have evaluated the thermal expansion coefficient in case of binary liquid mixtures at a specific temperature. Ali et al. [21] calculated the thermal expansivity and isothermal compressibility of binary liquid mixtures at $298.15 \mathrm{~K}$ using hard-sphere models. Excess molar volumes and excess thermal expansivities of aqueous binary solutions of dimethyl sulfoxide (DMSO), tetrahydrofuran and 1,4-dioxane have been calculated by Saleh et al. [22]. Zarei and Jalili [23] measured densities of the binary mixtures and evaluated thermal expansion coefficient $(\alpha)$ and their excess values with excess molar volumes.

The study of dilute solutions helps to minimize the effect of viscosity, internal field etc., therefore, for dilution apolar solvent like $\mathrm{CCl}_{4}$ is used. Apolar solvent provides a medium and dilution for the mixture which, in turn, also minimizes the requirement of pure liquids in large quantity. The system studied here is almost pseudo-binary with concentrations of phenol varying between 0.013 and 0.016 and o-cresol varying be- 
tween 0.011 and 0.013. Both, DMSO and phenol/o-cresol have their wide applications in industrial and medicinal fields. DMSO is a high energy reaction solvent. Its cryoprotective effects on biological systems are well known. The highly polar $\mathrm{S}=\mathrm{O}$ group and two hydrophobic $-\mathrm{CH}_{3}$ group make it a polyfunctional molecule. Its polarity accelerates reactions with charged transition states, such as $S_{N} 2$ alkylations of phenols and indoles. Therefore, interesting results may be obtained regarding interaction study in ternary liquid mixtures of DMSO with phenol/o-cresol in carbon tetrachloride because DMSO provides $-\mathrm{S}=\mathrm{O}$ group and phenol/o-cresol provides $-\mathrm{OH}$ group for interactions. In the present communication, we have evaluated thermal expansivity using seven hard-sphere models viz., Thiele-Lebowitz, Thiele, CarnhanStarling, Guggenheim, scaled-particle theory, Henderson and Hoover-Ree and Flory's statistical theory for (a) $\mathrm{DMSO}+$ phenol $+\mathrm{CCl}_{4}$ and (b) DMSO + o-cresol $+\mathrm{CCl}_{4}$ at $293.15,303.15$ and $313.15 \mathrm{~K}$. The theoretical values of thermal expansivity are compared with the experimentally obtained values [24]. Apart from these, the excess thermal expansivity $\alpha^{\mathrm{E}}$ for the aforementioned mixtures has also been computed and molecular interactions among the participating molecules have been discussed.

\section{Theory}

\subsection{Hard-sphere models}

On the basis of seven hard-sphere models, thermal expansion coefficient of the liquid mixtures can be calculated theoretically by using the rigid-sphere equation of state as $[21,25]$ :

Thiele-Lebowitz model (TL):

$$
\alpha=\frac{1}{T} \frac{1-y^{3}}{(1+2 y)^{2}} .
$$

Thiele model $(\mathrm{T})$ :

$$
\alpha=\frac{1}{T} \frac{\left(1+2 y+3 y^{2}\right)(1-y)}{\left(1+5 y+9 y^{2}-3 y^{3}\right)} .
$$

Carnhan-Starling model (CS):

$$
\alpha=\frac{1}{T} \frac{\left(1+y+y^{2}-3 y^{3}\right)(1-y)}{\left(1+4 y+4 y^{2}-4 y^{3}+y^{4}\right)} .
$$

Guggenheim model $(\mathrm{G})$ :

$$
\alpha=\frac{1}{T} \frac{1-y}{1+3 y} .
$$

Scaled-particle theory model (ST):

$$
\alpha=\frac{1}{T} \frac{1-y}{1+y} .
$$

Henderson model $(\mathrm{H})$ :

$$
\alpha=\frac{1}{T} \frac{\left(8+y^{2}\right)(1-y)}{\left(8+8 y+3 y^{2}-y^{3}\right)} .
$$

Hoover-Ree model (HR):

$$
\alpha=\frac{1}{T} \frac{1+4 y+10 y^{2}+18.36 y^{3}+28.2 y^{4}+39.5 y^{5}}{1+8 y+30 y^{2}+73.44 y^{3}+141 y^{4}+237 y^{5}} .
$$

where $y$ denotes the packing fraction defined as;

$$
y=\frac{\pi d^{3} N}{6 V},
$$

where $d$ is the rigid-sphere diameter of liquid mixtures given as:

$$
d=(2 r) \text {. }
$$

$r$ is the molecular radius of liquid mixture [26] given as

$$
r=\sqrt[3]{\frac{M}{\rho N}} \sqrt[3]{\frac{3}{16 \pi}\left[1-\frac{\gamma R T}{M u^{2}}\left(\sqrt{1+\frac{M u^{2}}{\gamma R T}}-1\right)\right]},
$$

where $M$ is the molecular mass, $\rho$ is the density, $\gamma$ is the specific heat ratio, $u$ is the ultrasonic velocity in the liquid mixture, $R$ is the gas constant, $T$ is the temperature in $\mathrm{K}$. $N=6.023 \times 10^{23} \mathrm{~mol}^{-1}$ is the Avogadro number and $V$ is the molar volume of the mixture.

\subsection{Flory statistical theory}

The expression of thermal expansivity obtained from Flory's statistical theory [10-13] is given by

$$
\alpha=\frac{3\left(\tilde{V}^{1 / 3}-1\right)}{T\left\{\left[1-3\left(\tilde{V}^{1 / 3}-1\right)\right]\right\}},
$$

where $\tilde{V}$ is the reduced volume of the liquid.

Starting with the reduced equation of state gives

$$
\frac{\tilde{P} \tilde{V}}{\tilde{T}}=\frac{\tilde{V}^{1 / 3}}{\tilde{V}^{1 / 3}-1}-\frac{1}{\tilde{V} \tilde{T}} .
$$

The values of $\tilde{V}, V^{*}, P^{*}, \tilde{T}$ and $T^{*}$ of pure components can be obtained from the knowledge of thermal expansivity $(\alpha)$, isothermal compressibility $\left(\beta_{T}\right)$ and molar volume $(V)$ of the pure components. Segment fraction $(\Psi)$, site fraction $(\theta)$ interaction parameter $\left(X_{i j}\right)$, characteristic temperature $\left(T^{*}\right)$, characteristic pressure $\left(P^{*}\right)$ and the reduced temperature $(\tilde{T})$ of the liquid mixture have been computed by the earlier suggested methods [10-13]:

$$
\tilde{V}^{0}=\sum_{i=1}^{3} x_{i} \tilde{V}_{i}
$$

where $x_{i}$ and $\tilde{V}_{i}$ are the corresponding mole fractions and reduced volumes of $i$-th component.

$$
\begin{aligned}
& \tilde{T}^{0}=\frac{\left(\tilde{V}^{0^{1 / 3}}-1\right)}{\left(\tilde{V}^{0^{4 / 3}}\right)}, \\
& \tilde{V}^{\mathrm{E}}=\tilde{V}^{0^{7 / 3}}\left(4 / 3-\tilde{V}^{0^{1 / 3}}\right)^{-1}\left(\tilde{T}-\tilde{T}^{0}\right), \\
& \tilde{V}=\tilde{V}^{\mathrm{E}}+\tilde{V}^{0} .
\end{aligned}
$$

The values of $\alpha$ are obtained from Eq. (8), using the values of $\tilde{V}$ obtained from Eq. (12). The excess function $\alpha^{\mathrm{E}}$ is calculated as

$$
\alpha^{\mathrm{E}}=\alpha_{\text {mix }}-\alpha_{\text {ideal }},
$$

where $\alpha_{\text {mix }}$ is experimental value and $\alpha_{\text {ideal }}$ is the theoretical value of expansivity obtained by the relation

$$
\alpha_{\text {ideal }}=\sum_{i=1}^{3} \phi_{i} \alpha_{i}
$$


where $\alpha_{i}$ is the thermal expansion coefficient of the $i$-th component and volume fraction is given as

$$
\phi_{i}=\frac{\left(x_{i} V_{i}\right)}{\sum_{j=1}^{3} x_{j} V_{j}} .
$$

\section{Results and discussion}

Thermal expansivity $\alpha$ of ternary liquid mixtures consisting of DMSO with phenol/o-cresol in carbon tetrachloride have been computed by employing seven hard-sphere models and Flory's statistical theory at 293.15, 303.15 and $313.15 \mathrm{~K}$, respectively. The parameters of

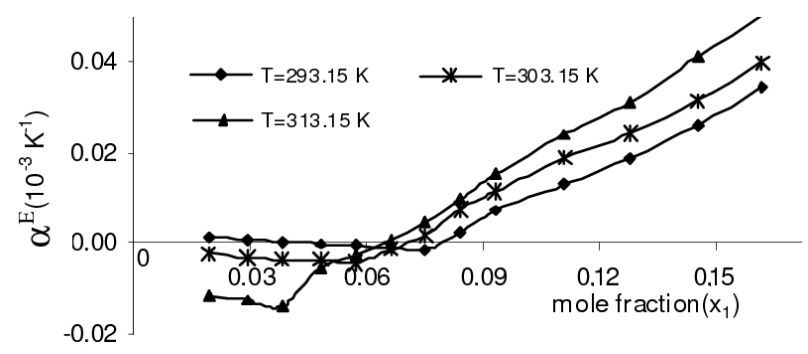

Fig. 1. A plot of excess thermal expansivity $\left(\alpha^{\mathrm{E}}\right)$ versus mole fraction for DMSO with phenol in carbon tetrachloride.

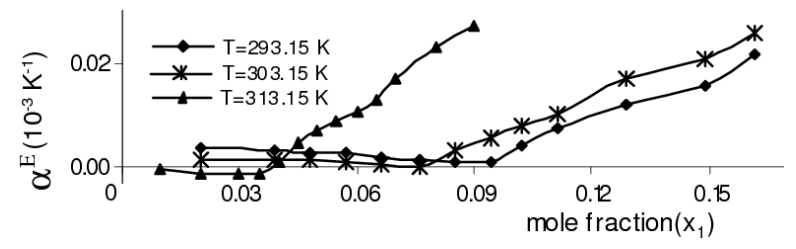

Fig. 2. A plot of excess thermal expansivity $\left(\alpha^{\mathrm{E}}\right)$ versus mole fraction for DMSO with o-cresol in carbon tetrachloride.

pure components are listed in Table I. Experimental and theoretically estimated values of thermal expansion coefficients for both ternary liquid systems are listed in Tables II and III. A plot of excess thermal expansivity $\left(\alpha^{\mathrm{E}}\right)$ as a function of concentration $\left(x_{1}\right)$ for both ternary systems are presented in Figs. 1 and 2. For the computation of thermal expansivity by Flory's statistical theory, the desired characteristic and reduced parameters have been computed by the procedure laid down in literature $[10-13,26,27]$ and packing fraction $y$, which is required for the evaluation of $\alpha$ by hard-sphere models, has been attained by the method suggested by Pant et al. [28]. The required data for calculation were measured previously in our laboratory [29].

TABLE I

Properties of pure component liquids. Taken from Ref. [29].

\begin{tabular}{c|c|c|c|c|c|c}
\hline \hline Components & $\begin{array}{c}\text { Temp. } \\
{[\mathrm{K}]}\end{array}$ & $\begin{array}{c}\alpha\left(\times 10^{-3}\right) \\
{\left[\mathrm{K}^{-1}\right]}\end{array}$ & $\tilde{T}$ & $\begin{array}{c}T^{*} \\
{[\mathrm{~K}]}\end{array}$ & $\begin{array}{c}V^{*} \\
{\left[\mathrm{~cm}^{3} / \mathrm{mole}\right]}\end{array}$ & $\begin{array}{c}P *\left(\times 10^{9}\right) \\
{\left[\text { dyne } / \mathrm{cm}^{2}\right]}\end{array}$ \\
\hline \multirow{2}{*}{ DMSO } & 293.15 & 0.975 & 0.056 & 5265.9 & 57.382 & 9.28 \\
& 303.15 & 0.979 & 0.057 & 5333.5 & 57.301 & 9.60 \\
& 313.15 & 0.984 & 0.058 & 5394.9 & 57.368 & 9.87 \\
phenol & 293.15 & 1.012 & 0.057 & 5156.4 & 71.043 & 8.39 \\
& 303.15 & 1.020 & 0.058 & 5214.9 & 71.114 & 8.60 \\
$\mathrm{CCl}_{4}$ & 313.15 & 1.031 & 0.060 & 5264.2 & 71.084 & 8.74 \\
& 293.15 & 1.132 & 0.060 & 4857.4 & 76.273 & 6.24 \\
& 303.15 & 1.145 & 0.062 & 4907.8 & 76.333 & 6.33 \\
o-cresol & 313.15 & 1.159 & 0.063 & 4956.4 & 76.689 & 6.39 \\
& 293.15 & 1.026 & 0.057 & 5120.0 & 84.294 & 8.10 \\
& 303.15 & 1.034 & 0.059 & 5174.8 & 84.220 & 8.28 \\
& 313.15 & 1.043 & 0.060 & 5230.3 & 84.138 & 8.46
\end{tabular}


TABLE II

Experimental (from Ref. [29]) and theoretical values of thermal expansivity: $\mathrm{DMSO}+$ phenol $+\mathrm{CCl}_{4}$.

\begin{tabular}{|c|c|c|c|c|c|c|c|c|c|c|}
\hline \multirow{2}{*}{$\begin{array}{c}x_{1} \\
\text { Mole frac. } \\
\text { of DMSO }\end{array}$} & \multirow{2}{*}{$\begin{array}{c}x_{3} \\
\text { Mole frac. } \\
\text { of } \mathrm{CCl}_{4}\end{array}$} & \multicolumn{9}{|c|}{$\alpha \times 10^{3}\left[\mathrm{~K}^{-1}\right]$} \\
\hline & & Exp. & TL model & $\mathrm{T}$ model & CS model & G model & ST model & $\mathrm{H}$ model & HR model & Flory \\
\hline \multicolumn{11}{|c|}{$293.15 \mathrm{~K}$} \\
\hline 1.000 & 0.000 & - & 1.557 & 1.620 & 1.545 & 1.529 & 2.112 & 2.094 & 1.593 & 0.975 \\
\hline 0.000 & 0.000 & - & 1.555 & 1.618 & 1.543 & 1.528 & 2.110 & 2.092 & 1.591 & 1.012 \\
\hline 0.000 & 1.000 & - & 1.565 & 1.627 & 1.553 & 1.538 & 2.120 & 2.102 & 1.600 & 1.132 \\
\hline 0.019 & 0.965 & 1.129 & 1.582 & 1.643 & 1.571 & 1.555 & 2.137 & 2.119 & 1.615 & 1.128 \\
\hline 0.029 & 0.956 & 1.127 & 1.577 & 1.639 & 1.566 & 1.551 & 2.132 & 2.115 & 1.611 & 1.126 \\
\hline 0.038 & 0.947 & 1.125 & 1.573 & 1.634 & 1.561 & 1.546 & 2.127 & 2.110 & 1.607 & 1.125 \\
\hline 0.048 & 0.937 & 1.124 & 1.568 & 1.630 & 1.556 & 1.540 & 2.122 & 2.105 & 1.602 & 1.124 \\
\hline 0.057 & 0.928 & 1.122 & 1.561 & 1.624 & 1.550 & 1.534 & 2.116 & 2.099 & 1.597 & 1.122 \\
\hline 0.066 & 0.919 & 1.120 & 1.558 & 1.621 & 1.546 & 1.530 & 2.113 & 2.095 & 1.593 & 1.121 \\
\hline 0.075 & 0.910 & 1.119 & 1.554 & 1.617 & 1.542 & 1.526 & 2.109 & 2.091 & 1.590 & 1.120 \\
\hline 0.084 & 0.902 & 1.122 & 1.555 & 1.618 & 1.543 & 1.527 & 2.110 & 2.092 & 1.590 & 1.120 \\
\hline 0.093 & 0.893 & 1.125 & 1.556 & 1.619 & 1.544 & 1.528 & 2.111 & 2.093 & 1.592 & 1.120 \\
\hline 0.111 & 0.875 & 1.129 & 1.552 & 1.616 & 1.540 & 1.524 & 2.107 & 2.089 & 1.588 & 1.118 \\
\hline 0.128 & 0.858 & 1.133 & 1.551 & 1.615 & 1.539 & 1.524 & 2.106 & 2.088 & 1.587 & 1.118 \\
\hline 0.145 & 0.842 & 1.138 & 1.556 & 1.619 & 1.544 & 1.528 & 2.111 & 2.093 & 1.591 & 1.117 \\
\hline 0.162 & 0.825 & 1.143 & 1.559 & 1.622 & 1.547 & 1.531 & 2.114 & 2.096 & 1.594 & 1.117 \\
\hline \multicolumn{11}{|c|}{$303.15 \mathrm{~K}$} \\
\hline 1.000 & 0.000 & - & 1.507 & 1.568 & 1.495 & 1.480 & 2.043 & 2.026 & 1.541 & 0.979 \\
\hline 0.000 & 0.000 & - & 1.505 & 1.566 & 1.494 & 1.479 & 2.042 & 2.025 & 1.540 & 1.020 \\
\hline 0.000 & 1.000 & - & 1.515 & 1.575 & 1.504 & 1.489 & 2.052 & 2.035 & 1.549 & 1.145 \\
\hline 0.019 & 0.965 & 1.138 & 1.532 & 1.590 & 1.521 & 1.506 & 2.068 & 2.051 & 1.564 & 1.140 \\
\hline 0.029 & 0.956 & 1.136 & 1.526 & 1.585 & 1.515 & 1.500 & 2.062 & 2.045 & 1.558 & 1.138 \\
\hline 0.038 & 0.947 & 1.135 & 1.521 & 1.580 & 1.509 & 1.494 & 2.057 & 2.040 & 1.554 & 1.137 \\
\hline 0.048 & 0.937 & 1.133 & 1.517 & 1.577 & 1.506 & 1.491 & 2.054 & 2.036 & 1.550 & 1.136 \\
\hline 0.057 & 0.928 & 1.131 & 1.514 & 1.575 & 1.503 & 1.488 & 2.051 & 2.034 & 1.548 & 1.134 \\
\hline 0.066 & 0.919 & 1.133 & 1.511 & 1.572 & 1.500 & 1.485 & 2.048 & 2.030 & 1.545 & 1.134 \\
\hline 0.075 & 0.910 & 1.135 & 1.508 & 1.568 & 1.496 & 1.481 & 2.044 & 2.027 & 1.542 & 1.133 \\
\hline 0.084 & 0.902 & 1.139 & 1.509 & 1.570 & 1.498 & 1.483 & 2.046 & 2.029 & 1.543 & 1.133 \\
\hline 0.093 & 0.893 & 1.142 & 1.510 & 1.570 & 1.498 & 1.483 & 2.046 & 2.029 & 1.544 & 1.133 \\
\hline 0.111 & 0.875 & 1.147 & 1.507 & 1.568 & 1.495 & 1.480 & 2.044 & 2.026 & 1.541 & 1.132 \\
\hline 0.128 & 0.858 & 1.150 & 1.505 & 1.566 & 1.493 & 1.478 & 2.042 & 2.024 & 1.539 & 1.131 \\
\hline 0.145 & 0.842 & 1.155 & 1.507 & 1.568 & 1.496 & 1.481 & 2.044 & 2.027 & 1.541 & 1.131 \\
\hline 0.162 & 0.825 & 1.161 & 1.512 & 1.573 & 1.501 & 1.486 & 2.049 & 2.032 & 1.546 & 1.130 \\
\hline \multicolumn{11}{|c|}{$313.15 \mathrm{~K}$} \\
\hline 1.000 & 0.000 & - & 1.460 & 1.519 & 1.449 & 1.434 & 1.979 & 1.963 & 1.493 & 0.984 \\
\hline 0.000 & 0.000 & - & 1.459 & 1.518 & 1.448 & 1.433 & 1.978 & 1.962 & 1.492 & 1.031 \\
\hline 0.000 & 1.000 & - & 1.469 & 1.527 & 1.458 & 1.443 & 1.988 & 1.972 & 1.501 & 1.159 \\
\hline 0.019 & 0.965 & 1.143 & 1.483 & 1.539 & 1.472 & 1.458 & 2.002 & 1.985 & 1.514 & 1.152 \\
\hline 0.029 & 0.956 & 1.141 & 1.478 & 1.535 & 1.467 & 1.453 & 1.997 & 1.981 & 1.509 & 1.150 \\
\hline 0.038 & 0.947 & 1.138 & 1.472 & 1.530 & 1.461 & 1.447 & 1.991 & 1.975 & 1.504 & 1.149 \\
\hline 0.048 & 0.937 & 1.146 & 1.469 & 1.527 & 1.458 & 1.444 & 1.989 & 1.972 & 1.501 & 1.149 \\
\hline 0.057 & 0.928 & 1.147 & 1.466 & 1.524 & 1.455 & 1.440 & 1.985 & 1.969 & 1.498 & 1.149 \\
\hline 0.066 & 0.919 & 1.149 & 1.463 & 1.521 & 1.452 & 1.437 & 1.982 & 1.966 & 1.496 & 1.148 \\
\hline 0.075 & 0.910 & 1.152 & 1.460 & 1.518 & 1.448 & 1.434 & 1.979 & 1.962 & 1.493 & 1.148 \\
\hline 0.084 & 0.902 & 1.156 & 1.461 & 1.520 & 1.450 & 1.435 & 1.981 & 1.964 & 1.494 & 1.148 \\
\hline 0.093 & 0.893 & 1.160 & 1.462 & 1.520 & 1.451 & 1.436 & 1.981 & 1.965 & 1.495 & 1.148 \\
\hline 0.111 & 0.875 & 1.166 & 1.460 & 1.519 & 1.448 & 1.434 & 1.979 & 1.963 & 1.493 & 1.147 \\
\hline 0.128 & 0.858 & 1.171 & 1.457 & 1.516 & 1.446 & 1.431 & 1.977 & 1.960 & 1.491 & 1.147 \\
\hline 0.145 & 0.842 & 1.178 & 1.458 & 1.517 & 1.447 & 1.432 & 1.978 & 1.961 & 1.491 & 1.147 \\
\hline 0.162 & 0.825 & 1.185 & 1.462 & 1.521 & 1.451 & 1.436 & 1.982 & 1.965 & 1.495 & 1.146 \\
\hline
\end{tabular}


TABLE III

Experimental (from Ref. [29]) and theoretical values of thermal expansivity: $\mathrm{DMSO}+\mathrm{o}-\mathrm{cresol}+\mathrm{CCl}_{4}$.

\begin{tabular}{|c|c|c|c|c|c|c|c|c|c|c|}
\hline \multirow{2}{*}{$\begin{array}{c}x_{1} \\
\text { Mole frac. } \\
\text { of DMSO }\end{array}$} & \multirow{2}{*}{$\begin{array}{c}x_{3} \\
\text { Mole frac. } \\
\text { of } \mathrm{CCl}_{4}\end{array}$} & \multicolumn{9}{|c|}{$\alpha \times 10^{3}\left[\mathrm{~K}^{-1}\right]$} \\
\hline & & Exp. & TL model & $\mathrm{T}$ model & CS model & G model & ST model & $\mathrm{H}$ model & HR model & Flory \\
\hline \multicolumn{11}{|c|}{$293.15 \mathrm{~K}$} \\
\hline 1.000 & 0.000 & - & 1.557 & 1.620 & 1.545 & 1.529 & 2.112 & 2.094 & 1.593 & 0.975 \\
\hline 0.000 & 0.000 & - & 1.552 & 1.616 & 1.540 & 1.524 & 2.107 & 2.089 & 1.588 & 1.026 \\
\hline 0.000 & 1.000 & - & 1.565 & 1.627 & 1.553 & 1.538 & 2.120 & 2.102 & 1.600 & 1.132 \\
\hline 0.020 & 0.967 & 1.131 & 1.573 & 1.635 & 1.562 & 1.547 & 2.128 & 2.111 & 1.608 & 1.129 \\
\hline 0.039 & 0.948 & 1.129 & 1.567 & 1.629 & 1.555 & 1.540 & 2.122 & 2.104 & 1.602 & 1.126 \\
\hline 0.048 & 0.939 & 1.127 & 1.564 & 1.626 & 1.552 & 1.536 & 2.119 & 2.101 & 1.599 & 1.125 \\
\hline 0.057 & 0.930 & 1.126 & 1.560 & 1.623 & 1.548 & 1.533 & 2.115 & 2.097 & 1.596 & 1.123 \\
\hline 0.066 & 0.921 & 1.124 & 1.557 & 1.620 & 1.545 & 1.529 & 2.112 & 2.094 & 1.592 & 1.122 \\
\hline 0.076 & 0.912 & 1.122 & 1.554 & 1.617 & 1.541 & 1.526 & 2.109 & 2.091 & 1.589 & 1.121 \\
\hline 0.085 & 0.903 & 1.121 & 1.550 & 1.614 & 1.538 & 1.522 & 2.105 & 2.087 & 1.586 & 1.119 \\
\hline 0.094 & 0.894 & 1.119 & 1.547 & 1.611 & 1.534 & 1.519 & 2.102 & 2.084 & 1.583 & 1.118 \\
\hline 0.102 & 0.886 & 1.121 & 1.546 & 1.610 & 1.533 & 1.518 & 2.101 & 2.083 & 1.583 & 1.118 \\
\hline 0.111 & 0.877 & 1.124 & 1.546 & 1.610 & 1.533 & 1.518 & 2.101 & 2.083 & 1.582 & 1.117 \\
\hline 0.129 & 0.859 & 1.126 & 1.543 & 1.608 & 1.531 & 1.515 & 2.098 & 2.080 & 1.580 & 1.116 \\
\hline 0.146 & 0.842 & 1.127 & 1.542 & 1.606 & 1.529 & 1.514 & 2.097 & 2.079 & 1.579 & 1.115 \\
\hline 0.162 & 0.827 & 1.131 & 1.542 & 1.606 & 1.529 & 1.513 & 2.096 & 2.078 & 1.578 & 1.114 \\
\hline \multicolumn{11}{|c|}{$303.15 \mathrm{~K}$} \\
\hline 1.000 & 0.000 & - & 1.507 & 1.568 & 1.495 & 1.480 & 2.043 & 2.026 & 1.541 & 0.979 \\
\hline 0.000 & 0.000 & - & 1.503 & 1.564 & 1.491 & 1.476 & 2.039 & 2.022 & 1.537 & 1.034 \\
\hline 0.000 & 1.000 & - & 1.515 & 1.575 & 1.504 & 1.489 & 2.052 & 2.035 & 1.549 & 1.145 \\
\hline 0.020 & 0.967 & 1.142 & 1.525 & 1.584 & 1.514 & 1.499 & 2.061 & 2.044 & 1.557 & 1.141 \\
\hline 0.039 & 0.948 & 1.140 & 1.518 & 1.578 & 1.507 & 1.492 & 2.054 & 2.037 & 1.551 & 1.138 \\
\hline 0.048 & 0.939 & 1.138 & 1.515 & 1.575 & 1.503 & 1.488 & 2.051 & 2.034 & 1.548 & 1.137 \\
\hline 0.057 & 0.930 & 1.137 & 1.512 & 1.572 & 1.501 & 1.486 & 2.049 & 2.031 & 1.546 & 1.136 \\
\hline 0.066 & 0.921 & 1.135 & 1.509 & 1.570 & 1.498 & 1.483 & 2.046 & 2.029 & 1.543 & 1.134 \\
\hline 0.076 & 0.912 & 1.133 & 1.506 & 1.567 & 1.494 & 1.479 & 2.043 & 2.026 & 1.540 & 1.133 \\
\hline 0.085 & 0.903 & 1.135 & 1.503 & 1.565 & 1.492 & 1.477 & 2.040 & 2.023 & 1.538 & 1.132 \\
\hline 0.094 & 0.894 & 1.136 & 1.501 & 1.562 & 1.489 & 1.474 & 2.037 & 2.020 & 1.536 & 1.132 \\
\hline 0.102 & 0.886 & 1.138 & 1.500 & 1.561 & 1.488 & 1.473 & 2.036 & 2.019 & 1.534 & 1.131 \\
\hline 0.111 & 0.877 & 1.139 & 1.499 & 1.560 & 1.487 & 1.472 & 2.035 & 2.018 & 1.534 & 1.131 \\
\hline 0.129 & 0.859 & 1.144 & 1.496 & 1.558 & 1.484 & 1.469 & 2.033 & 2.016 & 1.532 & 1.130 \\
\hline 0.146 & 0.842 & 1.145 & 1.494 & 1.556 & 1.482 & 1.467 & 2.031 & 2.014 & 1.530 & 1.128 \\
\hline 0.162 & 0.827 & 1.148 & 1.493 & 1.555 & 1.481 & 1.466 & 2.029 & 2.012 & 1.528 & 1.127 \\
\hline \multicolumn{11}{|c|}{$313.15 \mathrm{~K}$} \\
\hline 1.000 & 0.000 & - & 1.460 & 1.519 & 1.449 & 1.434 & 1.979 & 1.963 & 1.493 & 0.984 \\
\hline 0.000 & 0.000 & - & 1.456 & 1.515 & 1.445 & 1.430 & 1.975 & 1.959 & 1.489 & 1.043 \\
\hline 0.000 & 1.000 & - & 1.469 & 1.527 & 1.458 & 1.443 & 1.988 & 1.972 & 1.501 & 1.159 \\
\hline 0.020 & 0.967 & 1.155 & 1.474 & 1.531 & 1.463 & 1.449 & 1.993 & 1.977 & 1.506 & 1.155 \\
\hline 0.039 & 0.948 & 1.151 & 1.468 & 1.526 & 1.457 & 1.443 & 1.987 & 1.971 & 1.500 & 1.152 \\
\hline 0.048 & 0.939 & 1.150 & 1.466 & 1.524 & 1.454 & 1.440 & 1.985 & 1.968 & 1.498 & 1.151 \\
\hline 0.057 & 0.930 & 1.148 & 1.463 & 1.522 & 1.452 & 1.437 & 1.982 & 1.966 & 1.496 & 1.149 \\
\hline 0.066 & 0.921 & 1.149 & 1.460 & 1.519 & 1.449 & 1.435 & 1.980 & 1.963 & 1.493 & 1.148 \\
\hline 0.076 & 0.912 & 1.152 & 1.458 & 1.517 & 1.447 & 1.432 & 1.978 & 1.961 & 1.491 & 1.148 \\
\hline 0.085 & 0.903 & 1.153 & 1.455 & 1.515 & 1.444 & 1.430 & 1.975 & 1.958 & 1.489 & 1.147 \\
\hline 0.094 & 0.894 & 1.154 & 1.454 & 1.513 & 1.442 & 1.427 & 1.973 & 1.956 & 1.487 & 1.147 \\
\hline
\end{tabular}




\subsection{Hard-sphere models}

A close perusal of Tables II and III reveals that the values of $\alpha$ evaluated by HS models are almost decreasing with increasing mole fractions of DMSO in both ternary liquid mixtures while experimentally observed values of $\alpha$ exhibit a non-linear trend showing strong molecular interactions among the components. The values of thermal expansivity, estimated using HS models are found to decrease with the increasing temperature. It is also observed that the best results are obtained by Guggenheim, Carnhan-Starling, Thiele-Lebowitz, Hoover-Ree, Thiele, Henderson and scaled-particle theory. Much higher values of $\alpha$ are obtained from $\mathrm{H}$ and ST models [21]. It can be conclusive to say that thermal expansivities obtained by hard-sphere models are significantly higher as compared with the experimental ones. Thus, in the present context these models are not appropriate to describe thermal expansion for the ternary mixtures under study.

\subsection{Flory theory}

From Tables II and III it is quite evident that expansivity almost decreases with increasing mole fractions of DMSO for both liquid mixtures. The values of thermal expansivity using the Flory theory and experimental values are found to increase with increasing temperature. Similar results were obtained by Pandey et al. [25]. It is observed from Tables II and III that values of thermal expansion coefficient obtained from Flory's statistical theory yield quite satisfactory results, which are in excellent agreement with the experimental values. The possible reason for this could be the fact that Flory theory utilizes the pure component values to determine the model parameters.

\subsection{Excess thermal expansivity $\left(\alpha^{\mathrm{E}}\right)$}

Excess thermal expansion coefficients $\alpha^{\mathrm{E}}$ have been neglected in interrelating thermodynamic properties, because they have usually been thought too small to be significant for thermodynamic considerations. The values of $\alpha^{\mathrm{E}}$ for non-polar mixtures are certainly small, and are of the order of $10^{-6} \mathrm{~K}^{-1}$, or less than $1 \%$ of the thermal expansivity coefficient $\alpha$ of mixtures. However, the values for polar mixtures are considerably larger, are of the order of $10^{-5} \mathrm{~K}^{-1}$, up to the order of $10^{-4} \mathrm{~K}^{-1}$ in extreme case, and more than a few percent of the total $\alpha$ of mixtures and significantly affect thermodynamic calculations. In the present investigation, $\alpha^{\mathrm{E}}$ values for both DMSO-phenol and DMSO-O-cresol are found to be of the order of $10^{-5}[30,31]$. The graphical display (Figs. 1 and 2) represents a non-linear behaviour of $\alpha^{\mathrm{E}}$ attributing to the presence of stronger intermolecular interactions between the unlike molecules than the like ones, in both the systems. At initial and final regions of the curve, both DMSO-phenol and DMSO-O-cresol systems, the effect of self-association dominates in contributing molecules which makes $\alpha^{\mathrm{E}}$ values positive. But, in the middle of the curve, the effect of hydrogen bonding between unlike molecules of phenol/o-cresol and DMSO, plays a significant role and reduces $\alpha^{\mathrm{E}}$ which corresponds to a dip in $\alpha^{\mathrm{E}}$ curve. The reduction of the number and size of microphases tends to make $\alpha^{\mathrm{E}}$ values negative. The increasingly negative $\alpha^{\mathrm{E}}$ values with the increase in temperature clearly indicate the enhanced molecular interaction between DMSO and phenol/o-cresol molecules. It is also seen that the temperature dependence of $\alpha^{\mathrm{E}}$ is small $[21,23,30]$.

\section{Conclusions}

The thermal expansion coefficient using hard-sphere models and Flory's statistical theory has been discussed. Since the systems are almost pseudo-binary therefore a slight change in the concentration of phenol/o-cresol may result in significant changes in the thermal expansivity coefficient up to a certain limit. The observed $\alpha^{\mathrm{E}}$ values for both the ternary mixtures indicate the formation of hydrogen bonding between $\mathrm{O}$-atom of DMSO molecule and $\mathrm{H}$-atom of phenol/o-cresol molecule.

\section{Acknowledgments}

We are grateful to the University Grants Commission, New Delhi for providing financial support (F. No. 33$-34 / 2007$ (SR)).

\section{References}

[1] E.J. Thiele, J. Chem. Phys. 39, 474 (1963).

[2] M.S. Wertheim, Phys. Rev. Lett. 10, 321 (1963).

[3] H. Reiss, H.L. Frisch, J.L. Lebowitz, J. Chem. Phys. 31, 369 (1959).

[4] E.A. Guggenheim, Mol. Phys. 9, 43 (1965).

[5] N.F. Carnhan, K.E. Starling, J. Chem. Phys. 51, 635 (1969).

[6] N.S. Snider, T.M. Herrington, J. Chem. Phys. 47, 2248 (1967).

[7] A. Mulero, C. Galan, F. Cuadros, Phys. Chem. Chem. Phys. 3, 4991 (2001).

[8] J.M. Bomont, J.L. Bretonnet, J. Chem. Phys. 121, 1548 (2004).

[9] M. Miandehy, H. Modarress, M.R. Dehghani, Fluid Phase Equilibria 239, 91 (2006).

[10] P.J. Flory, R.A. Orwoll, A. Vrij, J. Am. Chem. Soc. 86, 3507 (1964); 86, 3515 (1964).

[11] P.J. Flory, A. Abe, J. Am. Chem. Soc. 86, 3563 (1964).

[12] P.J. Flory, J. Am. Chem. Soc. 87, 1833 (1965).

[13] A. Abe, P.J. Flory, J. Am. Chem. Soc. 87, 1838 (1965).

[14] J.D. Pandey, R.K. Mishra, R. Dey, J. Mol. Liq. 123, 24 (2006).

[15] M. Gepert, E. Zorebski, A. Leszczynska, Fluid Phase Equilibria 233, 157 (2005). 
[16] S.L. Oswal, P. Oswal, R.L. Gardas, S.G. Patel, R.G. Shinde, Fluid Phase Equilibria 216, 33 (2004).

[17] B.K. Sharma, Indian J. Pure Appl. Phys. 25, 262 (1987).

[18] B.K. Sharma, J. Acoust. Soc. Am. 73, 106 (1983).

[19] B.K. Sharma, Acoust. Lett. 9, 101 (1986).

[20] V.A. Tabhane, S. Ghosh, S. Agarwal, J. Pure Appl. Ultrason. 21, 122 (1997).

[21] A. Ali, A.K. Nain, D. Chand, R. Ahmad, Phys. Chem. Liq. 43, 205 (2005).

[22] M.A. Saleh, S. Akhtar, M.S. Ahmed, M.H. Uddin, Phys. Chem. Liq. 40, 621 (2002).

[23] H.A. Zarei, F. Jalili, J. Chem. Thermodyn. 39, 55 (2007).
[24] J.D. Pandey, S.B. Tripathi, V. Sanghuri, J. Mol. Liq. 100, 153 (2002).

[25] J.D. Pandey, R. Dey, B.D. Bishtt, J. Mol. Liq. 111, 67 (2004).

[26] J.D. Pandey, N. Pant, J. Am. Chem. Soc. 104, 3299 (1982).

[27] J.D. Pandey, R.K. Shukla, A.K. Shukla, R.D. Rai, J. Chem. Soc. Faraday Trans. I 84, 853 (1988).

[28] N. Pant, C.V. Chaturvedi, C.D. Chaturvedi, Z. Phys. Chem. (Leipzig) 264, 513 (1983).

[29] A. Awasthi, J.P. Shukla, Ultrasonics 41, 477 (2003).

[30] K. Tamura, J. Thermal Anal. Cal. 57, 759 (1999).

[31] M. Nishimoto, K. Tamura, S. Murakami, J. Chem. Thermodyn. 29, 15 (1997). 